\title{
ACCORD: With Approximate Covering of Convex Orthogonal Decomposition
}

\author{
Mousumi Dutt ${ }^{1}$, Arindam Biswas ${ }^{1}$, and Partha Bhowmick ${ }^{2}$ \\ 1 Department of Information Technology, \\ Bengal Engineering and Science University, Shibpur, Howrah, India \\ \{duttmousumi, barindam\}@gmail .com \\ 2 Department of Computer Science and Engineering, \\ Indian Institute of Technology, Kharagpur, India \\ bhowmick@gmail.com
}

\begin{abstract}
A fast and efficient algorithm to obtain an orthogonally convex decomposition of a digital object is presented. The algorithm reports a sub-optimal solution and runs in $O(n \log n)$ time for a hole-free object whose boundary consists of $n$ pixels. The approximate/rough decomposition of the object is achieved by partitioning the inner cover (an orthogonal polygon) of the object into a set of orthogonal convex components. A set of rules is formulated based on the combinatorial cases and the decomposition is obtained by applying these rules while considering the concavities of the inner cover. Experimental results on different shapes have been presented to demonstrate the efficacy, elegance, and robustness of the proposed technique.
\end{abstract}

Keywords: convex component; convex decomposition; image analysis; polygon decomposition; rectangular component.

\section{Introduction}

Polygons are frequently used by practitioners to solve geometric problems related with image processing and computer vision [6, 7, 21 23, 27, 28]. Computationalgeometric problems on general polygons are solved usually by decomposing them into simpler components, solving the problem for each component using a specialized algorithm, and then combining the partial solutions. A polygon can be decomposed into rectangular components, convex components, star-shaped components, monotone components, etc. Polygon decomposition has many applications in theory and practice [4, 10, 25 27].

Polygon decompositions can be classified based on the interrelation among decomposed components. If the components do not overlap except at their boundaries, then the decomposition is termed as a partition. If overlapping pieces are allowed, then it is called a cover. Decomposing a polygon into simpler components can be performed with or without addition of extra vertices, which makes the problem complex but leads to fewer components. A decomposition must be minimal in some sense. Some applications use decomposition of a polygon into 


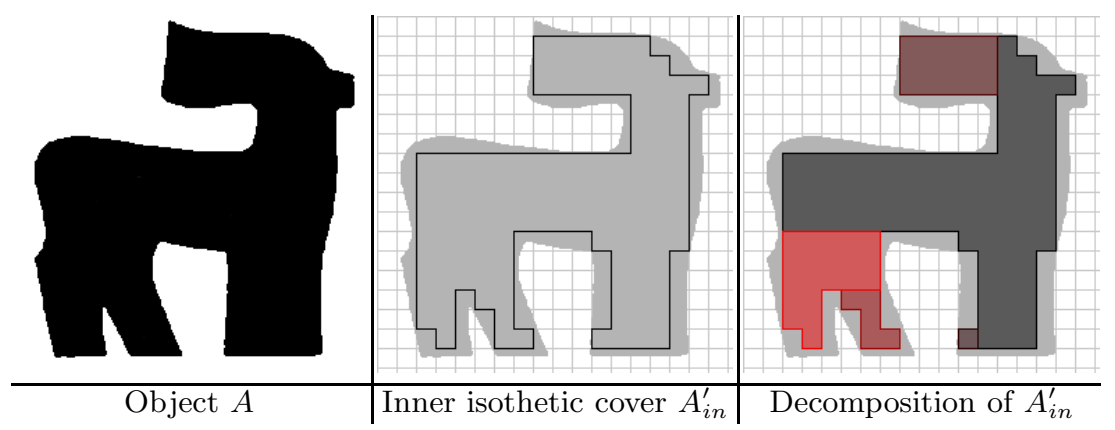

Fig. 1. A sample digital object and its orthogonal decomposition by our algorithm

minimum number of components, and some other applications use decomposition that minimizes the total length of internal edges [1]. In our work, decomposition is performed using the notion of partitioning.

Many works have been done on decomposition in general domain as well as in orthogonal domain. In general domain, decomposition of a polygon with holes into minimum number of components is NP-hard [5, 15, 17, 20], whether allowing or disallowing Steiner points. Allowing Steiner points, an $O(n \log n)$ time approximation algorithm under minimum edge-length criteria was stated in [12]. No optimal algorithm is known for decomposing hole-containing polygons when Steiner points are allowed; in [8, 16], it has been shown that such problems are NP-hard. In 3D domain also, several works have been done, and in 1, 13, 14] some approximation algorithms are given, since exact convex decomposition is NP-hard. Various applications of decomposition problems may be seen in $[2,7,16,18,19]$.

Interestingly, the problems which are NP-hard for general polygons, may become tractable when restricted to orthogonal domain. An $O\left(n^{2}\right)$-time algorithm to cover a simple orthogonal polygon with minimum number of orthogonally convex polygons is given in 24], where polygons are classified and decomposed based on dent diagrams. Another $O\left(n^{2}\right)$-time algorithm in [9] gives the solution to the problem of covering a horizontally convex orthogonal polygon with minimum number of orthogonally convex polygons and with the minimum number of orthogonal star-shaped polygons. But, our algorithm allows all kinds of orthogonal polygon without holes as input. Our work is focused on the decomposition of a given orthogonal polygon (i.e., polyomino) without holes (which acts as the tight inner isothetic cover of a hole-free digital object) into a sub-optima1 1 set of orthogonally convex components (OCC, or, $h v$-convex polyominoes) such that (c1) each OCC is orthogonal with all its vertices as grid points and (c2) no two OCC overlap each other except at their boundaries. Condition c1 implies that extra vertices are allowed only in the form of grid points. Figure 1 illustrates a digital object $A$, its inner isothetic cover (for $g=13$ ), and the decomposed

${ }^{1}$ Exhaustive experimentation (Sec. 4) shows that our algorithm frequently produces optimal solution. 


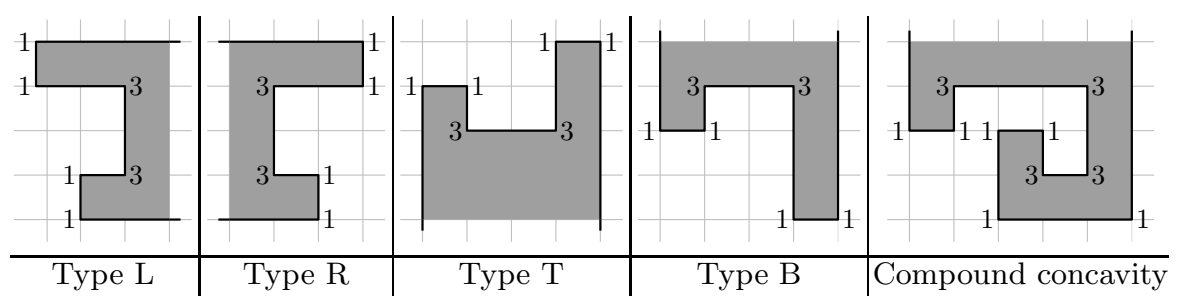

Fig. 2. Different types of concavities

set of OCC (which needed no extra vertex). It should be mentioned here that, to the best of our knowledge, there exists no proof till date to show whether partitioning an orthogonal polygon into a minimal set of OCCs can be done in polynomial time.

\section{Preliminaries}

A given digital object, $A$, is first imposed on an orthogonal grid, $G$. The grid consists of a set of equi-spaced horizontal lines and a set of equi-spaced vertical lines, their vertical/horizontal spacing being termed as the grid spacing, $g$. Using the algorithm TIPS [3], we obtain (the ordered set of vertices of) the inner isothetic cover, $A_{i n}^{\prime}$, which is the maximum-area orthogonal polygon inscribing $A$ such that the vertices of $A_{i n}^{\prime}$ are grid points (points of intersection among horizontal and vertical grid lines).

\subsection{Storing the Vertices}

The vertices of $A_{i n}^{\prime}$ are dynamically inserted in the circular doubly-linked list, $L$, and simultaneously in two temporary tables, $H_{x}$ and $H_{y}$, during the construction of $A_{i n}^{\prime}$. Each table is structured like a hash table without any hash key, and contains all slots from the minimum to the maximum grid coordinate. In $H_{x}$ $\left(H_{y}\right)$, a vertex $v_{i}\left(x_{i}, y_{i}\right)$, is stored in the slot of $x_{i}\left(y_{i}\right)$. The size of each slot $x_{i}$ $\left(y_{i}\right)$ of $H_{x}\left(H_{y}\right)$ is dynamically allocated depending on the number of vertices having abscissa $x_{i}$ (ordinate $y_{i}$ ); the concerned number of vertices is obtained by lexicographic sorting of $L$ to two temporary lists, $L_{x}^{\prime}(x$ - and $y$-coordinates as the respective primary and secondary keys) and $L_{y}^{\prime}(y$ - and $x$-coordinates as the respective primary and secondary keys). $L_{x}^{\prime}\left(L_{y}^{\prime}\right)$ is also used to prepare the slots of $H_{x}\left(H_{y}\right)$ in $O(n / g)$ time, since $O(n / g)$ is the number of vertices (explained in Sec. 3.5), $n$ being the number of border pixels of $A$, and $g$, the grid size. For each vertex $v_{i}, L$ contains its coordinates, type $(=1$ when the internal angle at $v_{i}$ is $\theta_{i}=90^{\circ}$ and 3 when $\theta_{i}=270^{\circ}$ ), and $i d$ of the cell incident at $v_{i}$ with partial object occupancy only if type $=3$; for type $=1$, id is set to 0 . (Example: With type $=3$, if the top-right cell is partially occupied by the object $A$, then $i d=1$; if it is the top-left one, then $i d=2$, and so on.)

In $L$, the component number $k_{i}$ (initialized as ' 1 ') corresponding to each vertex $v_{i}$, is also stored. When a component is extracted during decomposition, the component count $k$ is increased and $k_{i}$ of each vertex of the extracted 


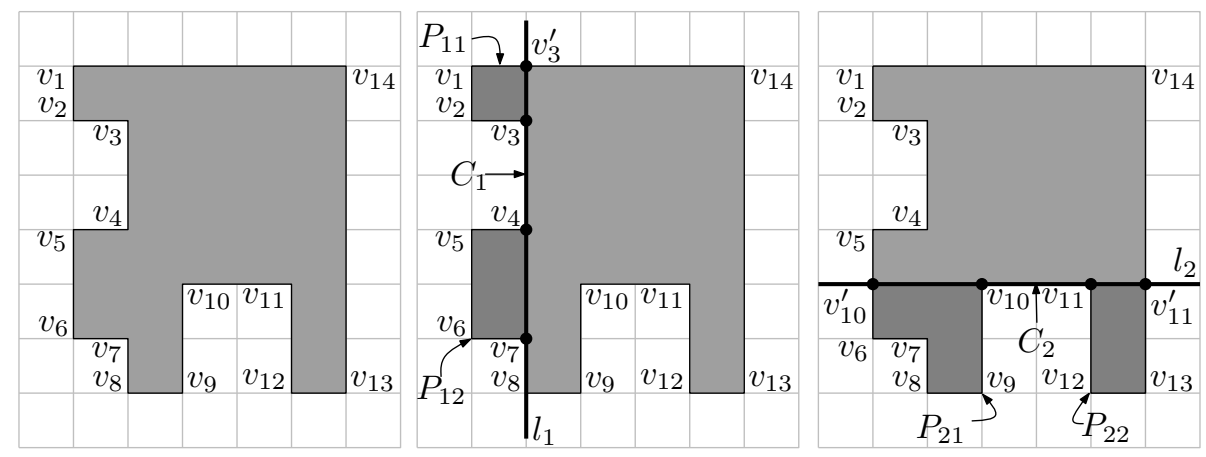

Fig. 3. Start and terminal vertices of $P_{11}, P_{12}, P_{21}, P_{22}$ w.r.t. $C_{1}$ and $C_{2}$

component is reset to $k$. During the construction of $A_{i n}^{\prime}$, a linked list $L_{c}$ is created, which contains all the concavities in the order as they appear in $L$. (Two or more consecutive Type 3 vertices creates a concavity [Fig. 2.) Each entry in $L_{c}$ contains the coordinates of two consecutive Type 3 vertices of the concerned concavity along with concavity type and concavity number. A vertex pattern of "1331" may occur in four possible simple concavities, namely, Type L (left), Type R (right), Type T (top), and Type B (bottom) [Fig. 2], determined from the Type 3 vertex $i d$ s stored in $L$. Three or more consecutive Type 3 vertices form a compound concavity, which are stored as simple concavities in $L_{c}$, each consisting of two consecutive Type 3 vertices and these simple concavities are assigned the same concavity number in $L_{c}$.

\subsection{Identifying the Sub-polygons of a Concavity}

If a horizontal/vertical line (as the case may be) $l_{i}$ is drawn along the (simple) concavity $C_{i}$, then the polygon is divided into two sub-polygons lying on one side of $l_{i}$ and the the main polygon on other side (Fig. 3). $l_{i}$ is called concavity line passing through the consecutive Type 3 vertices of $C_{i}$. If one of the two sub-polygons is extracted as an OCC, then $C_{i}$ is resolved. Each sub-polygon has at least two points on the concavity line, $l_{i}$, named as the start vertex (Type 3 ) and the terminal vertex. In Fig. 3, for concavity $C_{1}$, the respective sub-polygons $P_{11}$ and $P_{12}$ have $s_{11}=v_{3}$ and $s_{12}=v_{4}$ as start vertices, and $t_{11}=v_{3}^{\prime}$ and $t_{12}=v_{7}$ as terminal vertices. The sub-polygon appearing before (after) the concavity is described in the clockwise (counterclockwise) manner.

Terminal vertex of a sub-polygon is found using $H_{x}$ and $H_{y}$, as all vertical edges are stored in $H_{x}$ and horizontal edges in $H_{y}$. For the polygon shown in Fig. 3, $L=\left\langle v_{1}, v_{2}, \ldots, v_{14}\right\rangle$. For $C_{1}$ (Type L), we obtain the slot of $H_{x}$ containing the start vertices $v_{3}$ and $v_{4}$. As $v_{7}$ appears next to $v_{4}, v_{7}$ becomes the terminal vertex of $P_{12}$. However, as no vertex exists in $H_{x}$ before $v_{3}$, the horizontal edge $\left(v_{1}, v_{14}\right)$ lying above $v_{3}$ is detected from $H_{y}$. The $x$-coordinate of $v_{3}$ lies between those of $v_{1}$ and $v_{14}$, whence the $y$-coordinate of the terminal vertex of $P_{11}$ equals that of $v_{1}$ or $v_{14}$. For $C_{2}$ (Type B) or a Type $\mathrm{T} /$ Type $\mathrm{R}$ concavity, the terminal vertices are detected in a similar manner. 


\section{Rules for Decomposition}

The decomposition is carried out using $L_{c}$ (Sec. 2.1). To resolve each concavity, apparently one convex component should be extracted. For example, in Fig. 3 , extraction of $P_{11}$ resolves $C_{1}$ and extraction of $P_{21}$ resolves $C_{2}$, which, however, does not yield an optimal solution. Hence, rules are always applied to a pair of concavities at a time. The (sub-)optimality is obtained as explained next.

\subsection{Two Simple, Orthogonal, Consecutive Concavities}

If the lines of concavity $l_{1}$ and $l_{2}$, corresponding to $C_{1}$ and $C_{2}$, are orthogonal and intersect at $v$, and no sub-polygon of any concavity contains the other concavity in full, then extraction of only one sub-polygon resolves both $C_{1}$ and $C_{2}$ (Fig. 4)(ad)). The combined type of $C_{1}$ and $C_{2}$ may be LB, BR, RT, or TL. By traversing from $s_{12}$ to $v=t_{12}$, the component is extracted to resolve two concavities at a time. We have the following cases:

$-v \in\left\{s_{11}, s_{12}, s_{21}, s_{22}\right\}$ (Fig. 4(a)): $v$ is a vertex present in $L$.

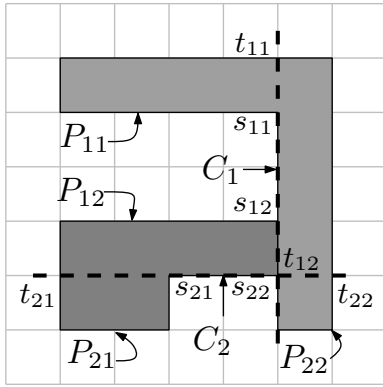

(a)

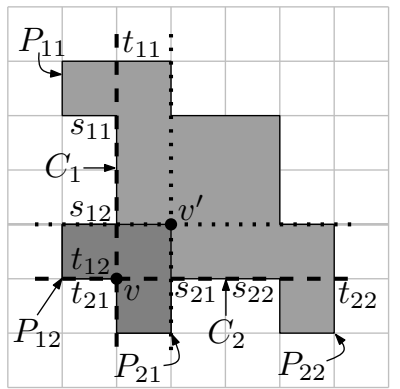

(d)

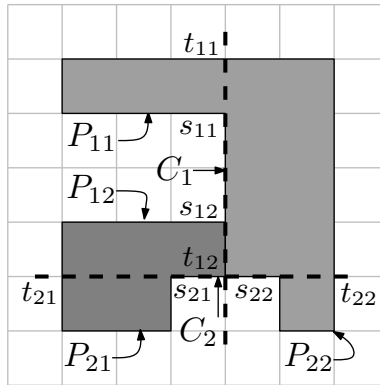

(b)

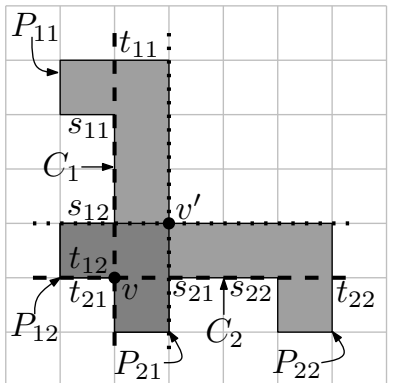

(e)

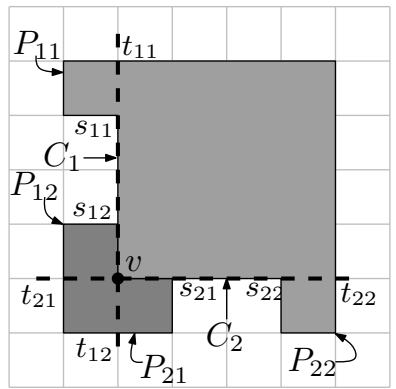

(c)

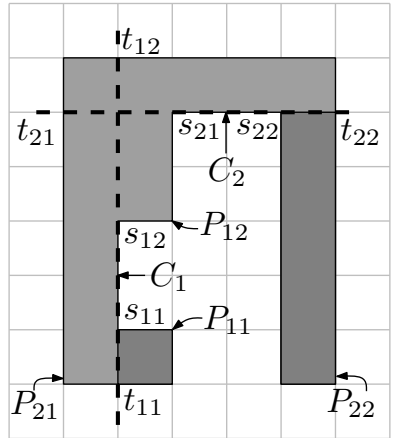

(f)

Fig. 4. Rules of decomposition for $l_{1} \perp l_{2}$. (There also exist rotational versions of the above cases - dealt in similar ways.) 
- $v$ lies on the edge $\left(s_{11}, s_{12}\right)$ or $\left(s_{21}, s_{22}\right)$ (Fig. 4(b)): $v$ is inserted appropriately in $L$ (using $H_{x}$ and $H_{y}$ ).

- $v$ lies not on the boundary but inside $A_{i n}^{\prime}$ (Fig. $\left.4(\mathrm{c})\right): v$ is inserted in $L$ (as above).

- $v$ lies on (the boundary of) or outside $A_{i n}^{\prime}$ (Fig. 4(d,e)): Find the point of intersection, $v^{\prime}$, between the line perpendicular to $l_{1}$ and passing through $s_{12}$ and the line perpendicular to $l_{2}$ and passing through $s_{21}$. If $v^{\prime}$ lies inside $A_{i n}^{\prime}$, then the component is extracted by anticlockwise traversal from $s_{12}$ down to $v^{\prime}$ and ending at $s_{12}$ (Fig. 4(d)). If $v^{\prime}$ lies on or outside $A_{i n}^{\prime}$, then $P_{11}$ is first extracted to solve $C_{1}$ and then $P_{22}$ to solve $C_{2}$ (Fig. 4(e)).

- If $C_{1}\left(C_{2}\right)$ lies entirely in one sub-polygon, say $P_{21}$, corresponding to $C_{2}$ $\left(C_{1}\right)$, then both $P_{11}$ and $P_{22}$ are extracted (Fig. 4(f)).

\subsection{Two Simple, Parallel, Consecutive Concavities}

If the projection of the edge $\left(s_{11}, s_{12}\right)$ on $l_{2}$ (or of the edge $\left(s_{21}, s_{22}\right)$ on $l_{1}$ ) lies on or inside $A_{i n}^{\prime}$, then extraction of one sub-polygon resolves both $C_{1}$ and $C_{2}$ (Fig. [5(a-d)). Otherwise, $P_{11}$ and $P_{21}$ are extracted to resolve the respective

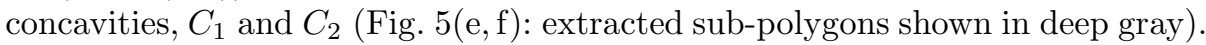

\subsection{Compound Concavities}

When there is a series of $t(>2)$ consecutive Type 3 vertices, then it is broken into $t-1$ simpler concavities, each consisting of two consecutive Type 3 vertices. For example, in Fig. 6, there are three pairs $L_{1} T_{1}, R_{1} B_{1}$, and $L_{2} T_{2}$, which are solved in three steps.

\subsection{Demonstration}

In Fig. [7(a), the concavities are numbered in the order in which they are stored in $L_{c}$. The first concavity in $L_{c}$ is $C_{1}$, whose next and previous concavities are $C_{2}$ and $C_{10}$, respectively. As the concavity lines of $C_{1}$ and $C_{10}$ are parallel, the rules of Sec. 3.2 are applied to extract the OCC (shown in deep gray in Fig. Z(c)). The second among the three consecutive Type 3 vertices (for $C_{1}$ and $C_{2}$ ) is included in the extracted OCC. Thus, three concavities are solved at a time $\left(C_{1}, C_{2}\right.$, and $\left.C_{10}\right) . L_{c}$ gets updated, and $C_{3}$ and $C_{4}$ are first checked, but no rules can be applied on them. So, $C_{3}$ is checked with its previous concavity in $L_{c}$, i.e., $C_{9}$. They are at $180^{\circ}$, and the OCC is extracted (Fig. $7(\mathrm{~d})$ ). Next pair is $C_{4}$ and $C_{5}$, which does not admit a single OCC extraction; so, $C_{4}$ is checked with its previous, i.e., $C_{8}$, but they also do not. Hence, one more backward traversal is made to test $C_{4}$ and $C_{7}$. Their concavity lines are parallel, and the OCC is extracted according to the rules stated in Sec. 3.2 (shown in Fig. 17(e)). In this manner, other concavities are also removed, to obtain the final result (Fig. 7 (f)). The total number of OCC is $k=7$ against the total number of simple concavities as $c=10$. 


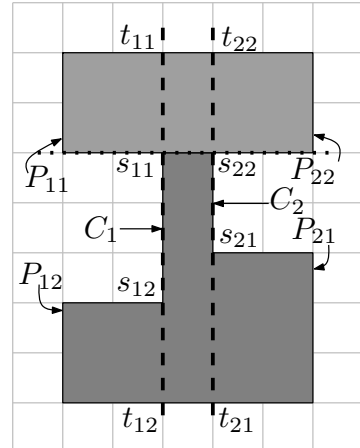

(a)

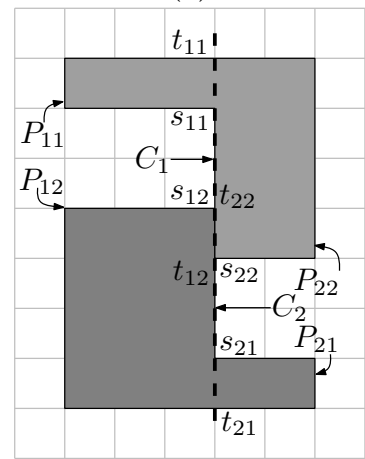

(d)

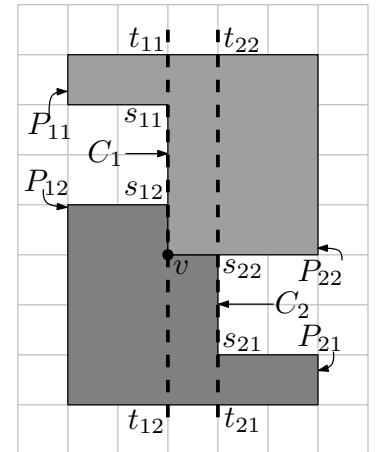

(b)

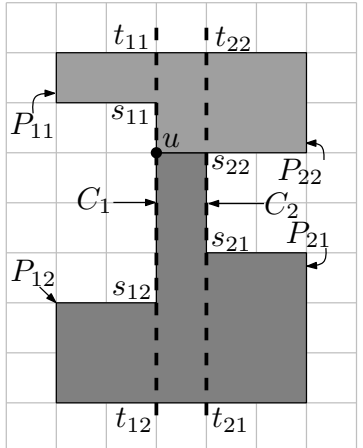

(c)

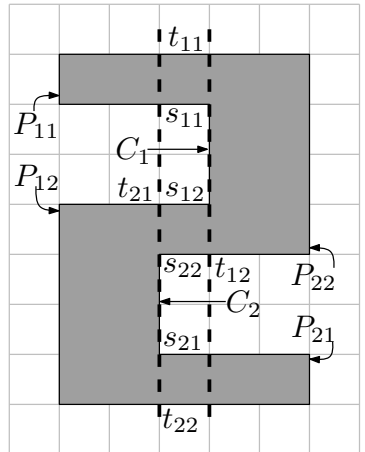

(f)

Fig. 5. Rules of decomposition for $l_{1}$ parallel to $l_{2}$. (There also exist rotational/flip versions of the above cases - dealt in similar ways.)

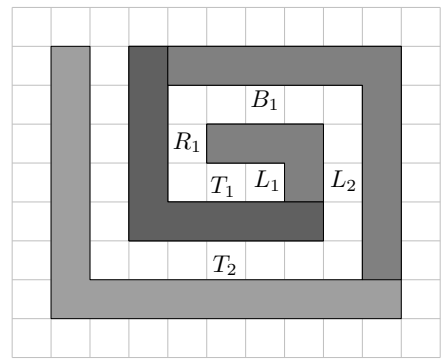

Fig. 6. Decomposition of a compound concavity

\subsection{Time Complexity}

The algorithm first constructs the inner isothetic cover $A_{i n}^{\prime}$, vertex list $L$, concavity list $L_{c}$, and the two hash tables $\left(H_{x}\right.$ and $\left.H_{y}\right)$. All this needs $O(n)$ time, 


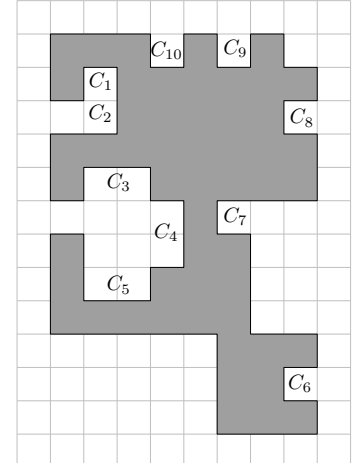

(a)

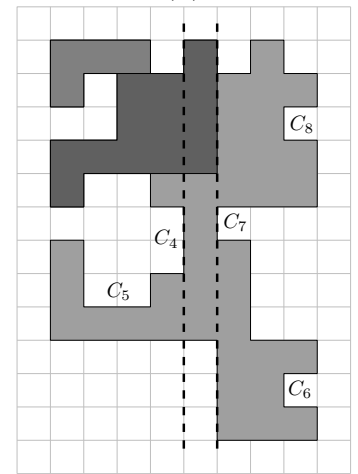

(d)

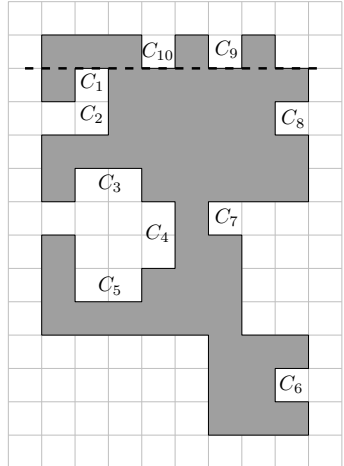

(b)

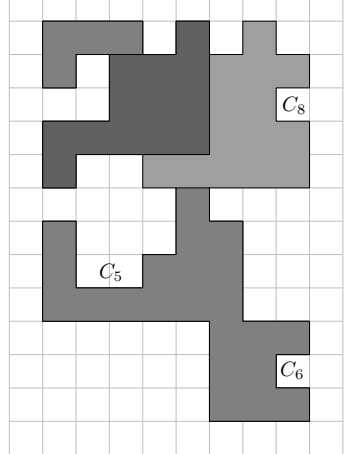

(e)

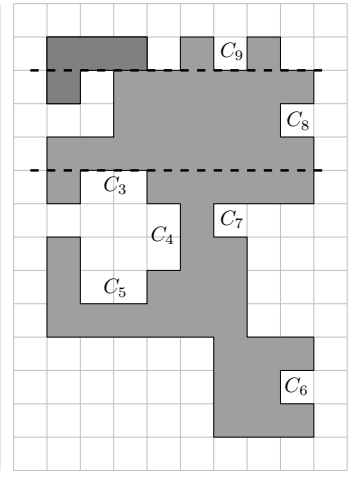

(c)

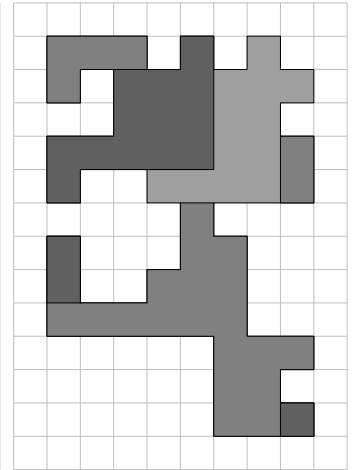

(f)

Fig. 7. A demonstration of getting OCC by our algorithm

where $n$ is the pixels constituting the contour of the object $A$, which is considered here as a connected component without holes. This follows from the combined fact that the intersection of each grid edge with $A$ is checked in $O(g)$ time, and the number of grid points visited is bounded by $O(n / g)$. All grid points lying on $A_{i n}^{\prime}$ are inserted in $L$ in $O(1)$ time. All the concavities are inserted in $L_{c}$ in $O(n)$ time. For preparation of $H_{x}$ and $H_{y}$ from $L_{x}^{\prime}$ and $L_{y}^{\prime}$ (Sec. 2.1), total computational cost is $O(n \log n)$. Hence, the total time complexity for Stage 1 is $O(n \log n)$.

In Stage 2, traversal of $L_{c}$ needs $O(n)$ time. Searching in $H_{x}$ and $H_{y}$ is performed in this stage, which takes $O(\log n)$ computational time per search. This follows from the fact that we apply binary search in each slot, which contains at most $O(n)$ elements in the dynamically-allocated array form (Sec. 2.1). There would be $O(n)$ such searches, consuming $O(n \log n)$ time in total. Insertion of extra grid points in $L$ may take $O(n \log n)$ time in worst case. Deletion of each node from $L_{c}$ requires $O(1)$ time, thereby consuming $O(n)$ time in total. Hence, the total worst-case time complexity for Stage 2 also is $O(n \log n)$, wherefore the overall time complexity of the entire algorithm becomes $O(n \log n)$. 


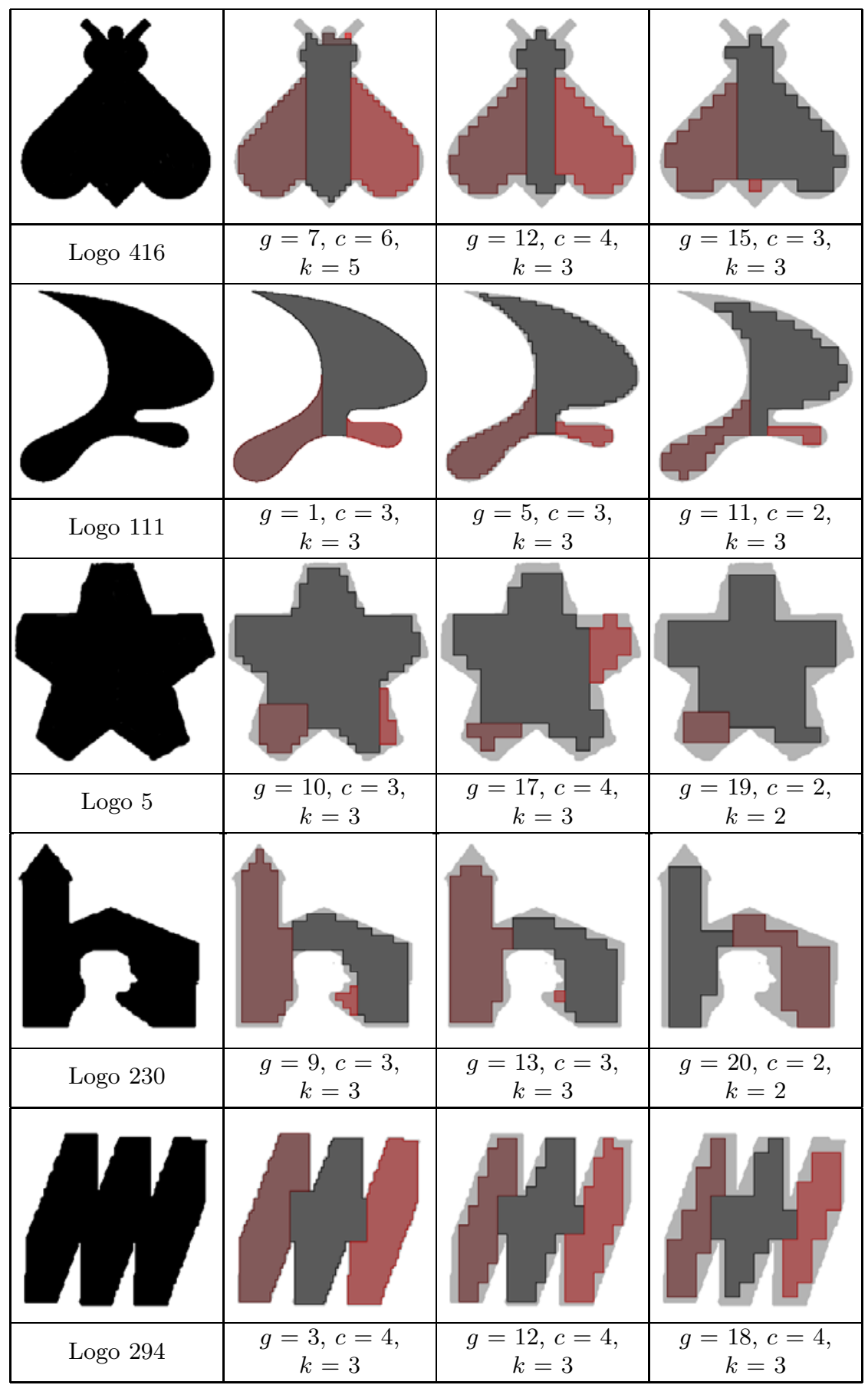

Fig. 8. Experimental results of orthogonal convex decomposition for various grid sizes 


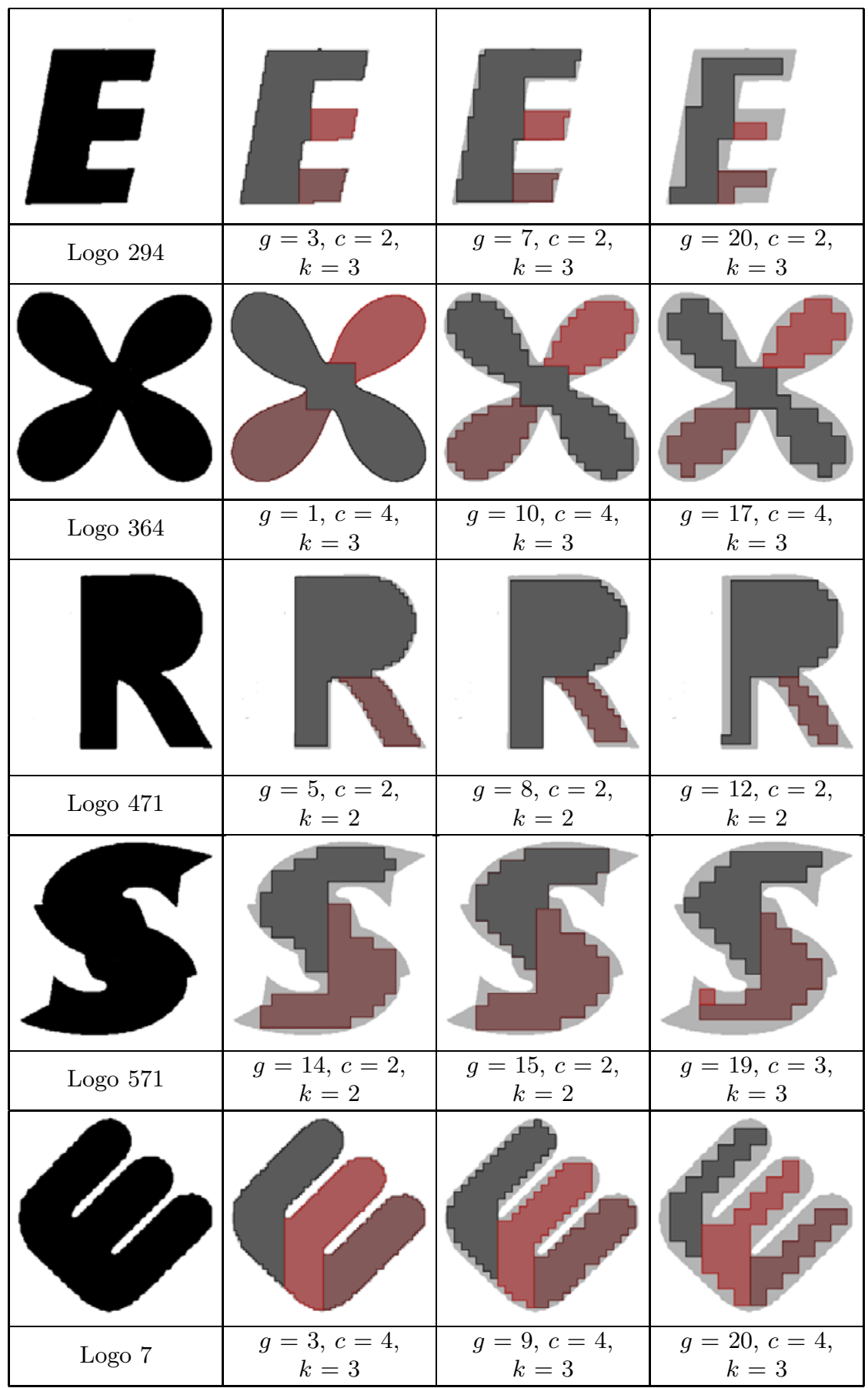

Fig. 9. Another set of results of orthogonal convex decomposition for various grid sizes 


\section{Results and Conclusion}

We have implemented the algorithm in $\mathrm{C}$ in Linux Fedora Release 7, Kernel version 2.6.21.1.3194.fc7, Dual Intel Xeon Processor $2.8 \mathrm{GHz}, 800 \mathrm{MHz}$ FSB. It is tested on several datasets containing various digital images of different shapes and forms. The decomposition takes place on inner cover of the images. In Figs. 8 and 9, results of several typical objects are given for various grid sizes. The different OCCs are marked by different colors. The total number of decomposed OCCs depends on the number of concavities and also on their mutual configurations and orientations. The count $k$ of OCC for a given digital object also depends on the grid size (required while extracting the inner cover $A_{i n}^{\prime}$ of the object $A$ ), since the number of concavities $(c)$ increases for lower grid sizes.

The proposed algorithm is designed in a manner so as to decompose arbitrary digital objects into orthogonally convex components (OCC), which might be useful for shape analysis. The algorithm can decompose a hole-free orthogonal polygon into a sub-optimal set of OCCs in $O(n \log n)$ time by considering all the concavities of the polygon. The steps of the algorithm are based on the rules designed out of the combinatorial possibilities. Exhaustive experimentation has been performed to verify its efficiency and robustness, and the results show the decompositions, which are mostly optimal.

\section{References}

1. Aguilera, A., Ayala, D.: Faster ASV Decomposition for Orthogonal Polyhedra, Using the Extreme Vertices Model (EVM). In: WSCG 2000, pp. 60-67 (2000)

2. Asano, T., Asano, T., Imai, H.: Partitioning a Polygonal Region into Trapezoids. JACM 33, 290-312 (1986)

3. Biswas, A., Bhowmick, P., Bhattacharya, B.B.: TIPS: On finding a tight isothetic polygonal shape covering a 2D object. In: Kalviainen, H., Parkkinen, J., Kaarna, A. (eds.) SCIA 2005. LNCS, vol. 3540, pp. 930-939. Springer, Heidelberg (2005)

4. Chazelle, B.: Approximation and Decomposition of Shapes. In: Schwartz, J.T., Yap, C.K. (eds.) Advances in Robotics 1: Algorithmic and Geometric Aspects of Robotics, pp. 145-186. Lawrence Erlbaum Associates, Hillsdale (1987)

5. Chazelle, B., Dobkin, D.: Decomposing a polygon into its convex parts. In: Proc. STOC, pp. 38-48 (1979)

6. Feng, H.Y.F., Pavlidis, T.: Decomposition of Polygons Into Simpler Components: Feature Generation for Syntactic Pattern Recognition. IEEE Trans. Computers 24, 636-650 (1975)

7. Ferrari, L., Sankar, P.V., Sklansky, J.: Minimal Rectangular Partitions of Digitized Blobs. CVGIP 28, 58-71 (1984)

8. Keil, J.M.: Decomposing a Polygon into Simpler Components. PhD thesis, Univ. of Toronto, Canada (1983)

9. Keil, J.M.: Minimally Covering a Horizontally Convex Orthogonal Polygon. In: Proc. SoCG, pp. 43-51 (1986)

10. Keil, J.M., Sack, J.R.: Minimum Decompositions of Polygonal Objects. In: Toussaint, J.T. (ed.) Computational Geometry, Netherlands, pp. 197-216 (1985) 
11. Klincsek, G.T.: Minimal Triangulations of Polygonal Domains. Discrete Math. 9, 121-123 (1980)

12. Levcopoulos, C., Lingas, A.: Bounds on the Length of Convex Partition of Polygons. In: Joseph, M., Shyamasundar, R.K. (eds.) FSTTCS 1984. LNCS, vol. 181, pp. 279-295. Springer, Heidelberg (1984)

13. Lien, J.-M., Amato, N.M.: Approximate convex decomposition of polygons. In: Proc. SoCG, pp. 17-26 (2004)

14. Lien, J.-M., Amato, N.M.: Approximate convex decomposition of polygons. CGTA 35, 100-123 (2006)

15. Lingas, A.: The power of non-rectilinear holes. In: Proc. ICALP. LNCS, vol. 140, pp. 369-383 (1982)

16. Lingas, A., Pinter, R., Rivest, R., Shamir, A.: Minimum Edge Length Partitioning of Rectilinear Polygons. In: Proc. 20th Allerton Conf. Commun. Control Comput., pp. 53-63 (1982)

17. Lingas, A., Soltan, V.: Minimum Convex Partition of a Polygon with Holes by Cuts in Given Directions. In: Proc. ISAAC, vol. 1178, pp. 315-325 (2006)

18. Nahar, S., Sahni, S.: Fast Algorithm for Polygon Decomposition. IEEE Trans. CAD 7, 473-483 (1988)

19. Ohtsuki, T.: Minimum Dissection of Rectiliniear Regions. In: Proc. IEEE Intl. Symp. Circuits \& Systems, pp. 1210-1213 (1982)

20. O'Rourke, J., Supowit, K.J.: Some NP-Hard Polygon Decomposition Problems. IEEE Trans. Information Theory 29, 181-190 (1983)

21. Pavlidis, T.: Shape Discrimination. In: Fu, K.S. (ed.) Syntactic Pattern Recognition, NY, pp. 125-145 (1977)

22. Pavlidis, T.: Structural Pattern Recognition. Springer, Berlin (1977)

23. Pavlidis, T.: Survey: A Review of Algorithms for Shape Analysis. CGIP 7, 243-258 (1978)

24. Reckhow, R.A., Culberson, J.: Covering a Simple Orthogonal Polygon with a Minimum Number of Orthgonally Convex Polygons. In: Proc. SoCG, pp. 43-51 (1986)

25. O'Rourke, J.: Art Gallery Theorems and Applications. Oxford University Press, NY (1987)

26. Shermer, T.C.: Recent Results in Art Gallery. Proc. of the IEEE 80(9), 1384-1399 (1992)

27. Toussaint, G.T.: Pattern Recognition and Geometrical Complexity. In: Proc. ICPR, pp. 1324-1347 (1980)

28. Waco, D.L., Kim, Y.S.: Geometric Reasoning for Matching Features Using Convex Decomposition. In: Proc. ACM Symp. Solid \& Physical Modeling, pp. 323-332 (1993) 